\title{
Die Impfung gegen Alzheimer - Ethische Fragen der Zukunft
}

\author{
Martina Schmidhuber (iD
}

Eingegangen: 10. Oktober 2019 / Angenommen: 31. Januar 2020 / Online publiziert: 24. Februar 2020 (C) Der/die Autor(en) 2020

Zusammenfassung 2018 waren weltweit 50Mio. Menschen von einer Demenz betroffen. Demenz ist sowohl aus sozialer als auch aus ökonomischer Sicht eine Herausforderung für die Gesellschaft. Ein Ziel der Alzheimer-Forschung ist u.a., einen Impfstoff gegen Alzheimer herzustellen. Bisher ohne Erfolg. Dennoch ist früher oder später ein Durchbruch zu erwarten. Welchen sozialen und ethischen Herausforderungen sich die Gesellschaft dann aber aufgrund der Impfung gegen Alzheimer stellen muss, wird im vorliegenden Text diskutiert. In Anlehnung an die aktuelle Impfdebatte werden Fragen zwischen Autonomie des Einzelnen und Verantwortung gegenüber der Gesellschaft betrachtet, sowie das Ziel einer Durchimpfungsrate und die damit möglicherweise verbundene Impfpflicht, das Recht auf Nicht-Wissen und das Recht auf Krankheit. Der Beitrag zeigt, dass eine AlzheimerImpfung eine Vielzahl von ethischen Herausforderungen implizieren würde.

Schlüsselwörter Alzheimer · Impfung · Autonomie · Gesellschaftliche Verantwortung $\cdot$ Recht auf Nicht-Wissen

\section{Vaccination against Alzheimer's disease—future ethical questions}

\begin{abstract}
Definition of the problem In 2018, 50 million people worldwide were affected by dementia. Dementia is a burden for society from both a social and economic perspective. One of the goals in Alzheimer's research is a vaccination against Alzheimer's disease, which unfortunately has not yet been successful. Nevertheless a breakthrough seems realistic and reachable.
\end{abstract}

Univ.-Prof. Dr. M. Schmidhuber $(\bowtie)$

Professur für Health Care Ethics, Universität Graz, Heinrichstraße 78b/1, 8020 Graz, Österreich

E-Mail: martina.schmidhuber@uni-graz.at 
Arguments The following article discusses the social and ethical challenges which a vaccination against Alzheimer's disease would implicate. Referring to the present debate on vaccination, the article considers the following aspects: autonomy of the individual, social responsibility, the goal of full vaccination coverage, mandatory vaccination, the right to ignorance and the right to illness.

Conclusion The article shows that a vaccination against Alzheimer's would be associated with many ethical challenges.

Keywords Alzheimer disease - Vaccination - Autonomy - Social responsibility · Right to ignorance

\section{Zukunftsmusik Alzheimer-Impfung}

Wir befinden uns im Jahr 2050 - Gesundheitsförderung spielt eine größere Rolle denn je. Auch hochaltrige Menschen leben gesund und ohne kognitive Einschränkungen. Einerseits deshalb, weil eine Reihe von medikamentösen und nicht-medikamentösen Interventionen gefunden wurde, welche kognitive Einschränkungen präventiv verhindern können, und andererseits, weil die Impfung gegen Alzheimer entdeckt wurde. Dank dieser medizinischen Errungenschaft gibt es keine an Alzheimer erkrankten Menschen mehr.

Wieder in der Gegenwart, müssen wir feststellen, dass aufgrund von noch nicht bewältigbaren Schwierigkeiten in der Alzheimer-Forschung in naher Zukunft noch kein Durchbruch in Form einer Impfung zu erwarten ist. Wir sind daher aufgerufen, weiterhin der ,,sozialen Dimension der Demenz“ Aufmerksamkeit zu widmen, wie der Soziologe Reimer Gronemyer postuliert (2013, S. 43). Mit welchen möglichen Herausforderungen aus ethischer und sozialer Sicht zu rechnen wäre, wenn es eines Tages die Alzheimer-Impfung geben soll, damit beschäftigt sich, in Anlehnung an die aktuelle Impfdebatte, der vorliegende Beitrag.

\section{Die Impfdebatte zwischen Autonomie und gesellschaftlicher Verantwortung}

Impfen ist zu einem hochsensiblen und emotionalen Thema in unserer Gesellschaft geworden. Vor allem neue Masernfälle haben die Debatte wieder entfacht. Laut WHO galten im Jahr 2017 die Masern in 37 von 53 Staaten der WHO-Region Europa als eliminiert. Im Mai 2019 allerdings warnte die WHO vor der Krankheit in dieser Region, denn innerhalb von 14 Monaten waren 90 Todesfälle zu beklagen (WHO 2019). Der Vorstoß im Jahr 2019 des amtierenden Gesundheitsministers Jens Spahn, die Masernimpfung verpflichtend einzuführen, löste viele Proteste aus. Da nicht alle Eltern bereit sind, ihre Kinder impfen zu lassen, sinkt die Durchimpfungsrate, was neue Fälle von Masern auftreten ließ; die Herdenimmunität ist nicht mehr gewährleistet. Bisher war in den Ländern Deutschland, Österreich und der Schweiz keine Impfung verpflichtend - die Pockenpflichtimpfung wurde 1982 in diesen Län- 
dern aufgehoben (Hirte 2012, S. 17). In Deutschland gibt die STIKO, die Ständige Impfkommission, lediglich Impfempfehlungen ab (Robert Koch Institut 2019).

Die Impfdebatte ist nicht zuletzt deshalb sehr emotional, weil hier zwei grundsätzliche ethische Prinzipien aufeinander prallen: Zum einen geht es um die Autonomie des Einzelnen - wer möchte Eltern aufoktroyieren, sein Kind impfen zu lassen, wenn sie es für gesünder und natürlicher halten, dies nicht zu tun und vielleicht sogar schon Kinder mit einem Impfschaden erlebt haben, sodass ihnen ihre ablehnende Haltung aufgrund dieses Einzelfalls scheinbar Recht gibt (Hirte 2012, S. 16)? Das Bundesverfassungsgericht erklärt, dass Eltern selbst entscheiden können, wie sie ihrer Verantwortung als Eltern gerecht werden wollen, frei von staatlichem Einfluss (Bundesverfassungsgericht, Urteil vom 16. Januar 2003 (2 BvR 716/01) in: BverfgE 107, 104 (117)). Zum anderen geht es aber um die Verantwortung, die man als Einzelner der Gesellschaft gegenüber hat. Ist das eigene Kind nicht geimpft, obwohl es schon geimpft sein könnte, ist es möglich, dass es bei Ausbruch der Masern Babys oder Menschen mit einem schwachen Immunsystem, die nicht geimpft werden können, ansteckt. Diese Ansteckung von Personen mit geschwächtem Immunsystem ist gefährlich und kann sogar zum Tode führen (Hirte 2012, S. 274; Deutscher Ethikrat 2019a).

Der Deutsche Ethikrat empfiehlt in seiner Stellungnahme vom Juni 2019 ,gezielte Informationskampagnen [...] um insbesondere bei Erwachsenen ein Bewusstsein für die Bedeutung eines Impfschutzes gegen Krankheiten $\mathrm{zu}$ schaffen, die viele fälschlich für Kinderkrankheiten halten“ (Deutscher Ethikrat 2019a, S. 85). Wenn eine Impfpflicht in Betracht gezogen werden sollte, dann dürfte sich diese lediglich auf einzelne Krankheiten beziehen. Das hätte wiederum zur Folge, dass Monopräparate zur Verfügung stehen müssten, was bisher in Deutschland nicht der Fall ist (Deutscher Ethikrat 2019a, S. 87).

Neben diesen aktuellen Fragen und Debatten zum Thema Impfen, ist in Zukunft noch mit weiteren Impfthemen zu rechnen; eines davon betrifft Alzheimer.

\section{Kommt die Impfung gegen Alzheimer?}

Forscher ${ }^{1}$ arbeiten schon seit vielen Jahren intensiv an der Suche nach einem Impfstoff gegen Alzheimer (Zeltins et al. 2017). Denn bis dato gibt es kein Mittel gegen die Alzheimer-Erkrankung, welche mit ca. $70 \%$ die häufigste Form der Demenzen darstellt. Demenzen werden in den nächsten Jahren laut World Alzheimer Report noch drastisch zunehmen: Alle drei Sekunden erkrankt ein Mensch an einer Demenz. Die Zahl von aktuell ca. 50 Mio. Menschen mit Demenz weltweit wird sich laut Experten-Prognosen 2050 mehr als verdreifacht haben (Alzheimer's Disease International 2018). Intensiv wird an den Ursachen und Gegenmitteln geforscht. Der größte Risikofaktor für eine Demenz ist das Alter: Ab 65 Jahren steigt das Risiko deutlich (Wallesch und Förstl 2017).

\footnotetext{
1 Aufgrund der besseren Lesbarkeit wird in diesem Beitrag das generische Maskulinum für beide Geschlechter verwendet.
} 
Wie genau Alzheimer entsteht und wie dagegen präventiv vorgegangen werden könnte, ist nach wie vor nicht völlig geklärt. Es wird davon ausgegangen, dass die Entstehung multifaktoriell ist, d. h. dass genetische, umweltbedingte und andere Faktoren zusammen Alzheimer verursachen. Erkennbar ist Alzheimer mittels MRT und CT an Ablagerungen von fehlgefalteten Proteinen (Amyloid- $\beta$ ), die sich zu harten, unauflöslichen Plaques anhäufen (Wallesch und Förstl 2017). Für eine Diagnose sind neben CT oder MRT weitere Screenings, Tests und eine Anamnese erforderlich. Bei Alzheimer handelt es sich um eine neurodegenerative Demenzform: Das Gehirn ist direkt erkrankt, die geschädigten Nervenzellen sind nicht mehr heilbar. Vermutet wird, dass die Schädigung der Nervenzellen bereits Jahre oder sogar Jahrzehnte vor der Diagnose beginnt (Wallesch und Förstl 2017). Was konkret präventiv gegen Alzheimer getan werden kann, ist nicht klar. Dennoch steht außer Frage, dass ein gesunder Lebensstil sowie geistige und körperliche Aktivität positiv wirken (Wallesch und Förstl 2017; Lenzen-Schulte 2018). Eine Garantie, nicht an Alzheimer zu erkranken, gibt es jedoch nicht. Menschen mit Alzheimer-Demenz werden aufgrund des Gedächtnisverlustes, der psychiatrischen Begleitsymptome, des Verlusts der Orientierung sowie schließlich des Verlusts der motorischen Fähigkeiten zum Pflegefall. Das stellt eine große Belastung für die Angehörigen dar, vor allem solange sie zu Hause betreuen und pflegen (Gräßel und Behrndt 2016). Darüber hinaus ist die Versorgung von Menschen mit Demenz aber auch mit einem hohen finanziellen Aufwand für die Gesellschaft verbunden. Im Jahr 2010 betrugen die Gesamtkosten für die Versorgung von Menschen mit Demenz weltweit 604 Mrd. US-Dollar (Wallesch und Förstl 2017; Alzheimer's Disease International 2010). Für das Jahr 2018 wurde eine Billion US-Dollar Kosten errechnet (Alzheimer's Disease International 2018, S. 34).

Kein Wunder also, dass sowohl aus menschlichen als auch aus ökonomischen Gründen intensiv nach einem Mittel gegen Alzheimer gesucht wird. Dennoch muss bedauerlicherweise konstatiert werden: „Antidementiva scheitern reihenweise. Kein Forschungszweig stagniert so sehr wie die Suche nach einem Mittel gegen Alzheimer" (Lenzen-Schulte 2018, A200). Seit 1998 wurden rund 100 Medikamente gegen Alzheimer getestet, erfolgreich waren lediglich vier (Alzheimer's Disease International 2018, S. 7). Vorübergehend können Antidementiva lediglich das Fortschreiten der Kernsymptome in bestimmten Phasen aufhalten. Psychiatrische Medikamente helfen gegen die psychiatrischen Begleitsymptome, allerdings können dadurch die demenziellen Symptome verstärkt werden.

Trotz Stagnation und Rückschlägen in der Forschung ist immer wieder von einer Impfung gegen Alzheimer die Rede. Und tatsächlich sind bereits positive Ergebnisse im Rahmen von Tierversuchen mit Mäusen zu verzeichnen, in denen die Amyloidbildung im Gehirn, die als maßgeblich für den geistigen Abbau gilt, verhindert, aufgehalten und sogar rückgängig gemacht werden konnte. Auf den Menschen war dies bisher nicht übertragbar. Klinische Studien mussten abgebrochen werden, weil es zu Entzündungen des Gehirns und der Hirnhäute kam (vgl. Zeltins et al. 2017).

Konkret hat die Wissenschaft zwei Ziele vor Augen: Bei der einen angestrebten Therapieform handelt es sich um Antikörpertherapien gegen Alzheimer bei bereits betroffenen Menschen. Die andere Form ist tatsächlich eine Impfung, die bei gesunden Menschen die Entstehung der Plaques im Gehirn verhindern soll. Dafür ist 
eine wichtige Voraussetzung, dass der Impfstoff eine starke Antikörperproduktion auslöst, die ausreichend ist, um die Plaqueablagerungen zu verhindern. Fest steht deshalb schon jetzt, dass eine Impfung bei Menschen, die bereits in einem frühen Stadium der Erkrankung sind, zu spät käme, sie müsste prophylaktisch angewandt werden (Bachmann et al. 2018).

Neben den erwähnten Schwierigkeiten im Rahmen der klinischen Studien ist die Amyloid-Theorie als Ursache für Alzheimer zudem nicht völlig unumstritten. Manche Forscher bezweifeln, dass es die Plaques sind, die zu Alzheimer führen (Tse und Herrup 2017). Da in die Forschung viel Geld für die Suche nach einem Impfstoff investiert wird, ist es wesentlich, sicher zu sein, wogegen die Alzheimer-Impfung wirken muss. Sind es nicht die Plaqueablagerungen, die verhindert werden müssen, um nicht an Alzheimer zu erkranken, ist das aktuelle Impfstoffkonzept obsolet.

Obwohl die Impfung gegen Alzheimer nicht zum Greifen nah scheint, ist es geboten, aus ethischer Sicht Überlegungen anzustellen, welche gesellschaftlichen und ethischen Konsequenzen die Impfung gegen Alzheimer hätte. Denn auch wenn diese Überlegungen angesichts der stagnierenden Forschung aktuell noch hypothetischer Natur sind, kann es als Aufgabe der Ethik betrachtet werden, schon im Vorhinein zu diskutieren, welche Konsequenzen ein solcher Durchbruch, der früher oder später stattfinden wird, hätte. Ähnlich verhält es sich bei den Debatten zu Gene Editing (Schmietow et al. 2019) oder zur Uterustransplantation (Bozzaro et al. 2019), in denen sich deutliche Entwicklungen und in diesen Fällen auch schon erste Erfolge zeigten. Es ist deshalb ein Gebot der Stunde, schon heute zu fragen, welche Folgen eine Impfung gegen Alzheimer morgen haben könnte und worauf es aus ethischer Sicht zu achten gilt. Die aktuelle, eingangs skizzierte Impfdebatte zur Impfpflicht zeigt bereits Tendenzen einer Richtung an, in die auch die Impfung gegen Alzheimer gehen könnte. Manches wird sich jedoch aufgrund der unterschiedlichen Ausgangslage anders darstellen: Alzheimer ist nicht ansteckend und die Impfung würde aller Voraussicht nach erwachsene Menschen und nicht Kinder, für die ihre Eltern entscheiden, betreffen.

Im Folgenden wird jedoch deutlich werden, dass es sich auch bei der AlzheimerImpfung, analog zur oben skizzierten Masern-Impfung, vor allem um das Spannungsfeld zwischen Autonomie des potenziellen Patienten und gesellschaftlicher Verantwortung handelt.

\section{Ethische Herausforderungen im Kontext einer Alzheimer-Impfung}

Ähnlich wie bei vielen anderen Erkrankungen, gegen die es Impfungen gibt, wird es sich voraussichtlich auch mit der Impfung gegen Alzheimer-Demenz verhalten: Alzheimer wird eines Tages Geschichte sein. Die gesellschaftlichen Herausforderungen im Umgang mit einer steigenden Zahl von Menschen mit verschiedenen Demenz-Formen sind enorm: Von der Überforderung der pflegenden Angehörigen über die adäquate Betreuung in Heimen bis hin zum Advance Care Planning (ACP) für den Fall einer Demenz, ist die Gesellschaft permanent gefordert, gute Lösungen zu finden. Demenzpläne und Demenzstrategien, empirische Sozialforschung zu/mit Menschen mit Demenz und ihren Angehörigen sowie neue Wohn- und Lebens- 
formen für Menschen mit Demenz - all diese Anstrengungen, um Menschen mit Demenz ein gutes Leben zu ermöglichen, wären leichter zu bewältigen, wenn die aktuell große Gruppe der Menschen mit Alzheimer aufgrund der Impfung nicht mehr erkrankt. Was aber gilt es auf dem Weg dorthin aus ethischer Perspektive zu berücksichtigen?

\section{Gesellschaftliche Verantwortung des Einzelnen}

Ist eine Impfpflicht eine geeignete Maßnahme, um Alzheimer in Zukunft gänzlich zu verhindern? Im Zuge der Pocken-Impfpflicht ist dies Anfang der 1980er-Jahre gelungen. Die Debatte, die im Alzheimer-Impf-Kontext entstehen könnte, lässt sich analog aus der aktuellen Impfpflichtdebatte übertragen: Sollte die Impfung gegen Alzheimer nicht verpflichtend sein, weil die individuelle Autonomie als hohes Gut gilt? Würden dann trotzdem jene als verantwortungslos betrachtet werden, die diese Impfung verweigern? Schon in den 1980er-Jahren postulierte die WHO in der Ottawa-Charta zur Gesundheitsförderung, dass alle Menschen dazu befähigt werden müssen, ihre Gesundheit zu stärken, indem ihnen ,ein höheres Maß an Selbstbestimmung über ihre Gesundheit" (WHO 1986) ermöglicht wird. Das impliziert den Zugang zu allen relevanten Informationen anhand derer alle Menschen selbst Entscheidungen in Bezug auf ihre persönliche Gesundheit treffen können (WHO 1986). Die Selbstbestimmung über persönliche Gesundheitsentscheidungen steht im Vordergrund. Dennoch könnten Menschen, welche die Impfung gegen Alzheimer für sich selbst ablehnen, als verantwortungslos angesehen werden, weil sie dem solidarischen Gesundheitssystem unnötige Mehr-Kosten verursachen. Ebenso könnte die Belastung für das persönliche Umfeld als Argument vorgebracht werden. Der Vorwurf der Verantwortungslosigkeit der Impfverweigerer würde sich also sehr wahrscheinlich auf die Kosten und die Belastung der Angehörigen beziehen und nicht, wie aktuell im Fall der Masernimpfung, auf die Ansteckung anderer Gesellschaftsmitglieder. Eine Analogie lässt sich am ehesten zu FSME ziehen: Auch hier wird geimpft, weil eine Wahrscheinlichkeit besteht, nach einem Zeckenstich an Meningitis oder Enzephalitis zu erkranken und bei ungünstigem Verlauf ein Pflegefall zu werden (Demicheli et al. 2009). Diese Impfung ist allerdings freiwillig.

Die Kosten für Betreuung und Pflege von Alzheimer-erkrankten Menschen wäre mit Sicherheit deutlich höher als jene für die Impfung. Bußgelder, wie sie auch aktuell vom deutschen Gesundheitsminister Spahn angedacht werden, könnten für die Impfverweigerer die Folge sein.

\section{Durchimpfungsrate}

Das führt zur Frage, wer denn überhaupt geimpft werden sollte. Ist das Ziel, Alzheimer komplett zu verhindern, wäre es angesichts der steigenden Anzahl von Betroffenen angezeigt, alle Menschen zu impfen. Nun stellt sich jedoch die Frage, ob möglicherweise mit Unverträglichkeiten der Impfung bei manchen Menschen zu rechnen wäre. Wie bereits erwähnt, gibt es im Rahmen der klinischen Studien gegenwärtig noch massive Schwierigkeiten mit entzündeten Hirnhäuten bei Probanden. Wird dieses Risiko auch bei der zugelassenen Impfung weiterhin bestehen, 
weil es Menschen gibt, deren Hirnhäute empfindlicher reagieren, z. B. jene, die als Kind bereits an einer Hirnhautentzündung litten? Wenn diese Art von Impfschaden auftreten sollte, wäre es mehr als bedenklich, eine Impfpflicht gegen Alzheimer einzuführen. Auch drängt sich die Frage auf, wer bei einem solchen Schaden finanziell aufkommen würde. Zurzeit fühlen sich Menschen, die aufgrund eines vermuteten Impfschadens beeinträchtigt sind, nicht entsprechend entschädigt (Zentrum der Gesundheit 2019).

Eine weitere Frage, die sich stellt, ist jene nach dem Alter: Ab welchem Alter wäre es erforderlich, sich gegen Alzheimer impfen zu lassen? Müsste dies erst getestet werden, würden die ersten Personen, die geimpft werden, noch das Risiko eingehen, trotz Impfung an Alzheimer zu erkranken, etwa weil das Impfalter zu spät festgelegt wurde. Hier stellt sich die Frage, wer die ersten sein sollten, die für die neu zugelassene Impfung zur Verfügung stehen. Wie müsste dies honoriert werden, da das Risiko - wie eben auch die aktuellen gescheiterten Studien zeigen - doch nicht unerheblich wäre? Und wie steht es um die Solidarität mit jenen, die zeitlich gerade noch nicht die Impfmöglichkeit erlebt haben und bereits erkrankt sind? Sie müssten freilich weiterhin gut versorgt werden.

Wäre es sinnvoll, bereits ab einem Lebensalter von 30 oder gar 20 Jahren alle Menschen zu impfen, weil damit nahezu garantiert würde, auch früh einsetzende Alzheimer (Early Onset Alzheimer) ausschließen zu können? Da im Rahmen der aktuellen Forschung vermutet wird, dass die Schädigung der Nervenzellen bereits Jahre oder sogar Jahrzehnte vor der Diagnose beginnt (Wallesch und Förstl 2017), wird diese Vorgehensweise sehr wahrscheinlich bedacht werden. Dies ist freilich eine Kostenfrage, weswegen vermutlich auch die Option in Erwägung gezogen würde, die Impfung nur bei Risikopersonen anzuwenden. Dafür wären wiederum Vortests erforderlich, die allerdings auch Kosten verursachen würden. Hier liegt nahe, dass eine Kosten-Abwägung durchgeführt würde, ob es für das Gesundheitssystem günstiger wäre, alle Menschen ab 30 Jahren verpflichtend zu impfen oder von allen Menschen ab 30 Jahren einen prädiktiven Test zu fordern. Beides ist jedoch ethisch problematisch: Die verpflichtende Impfung widerspricht dem Prinzip der Autonomie, die verpflichtenden Vortests widersprechen auch dem Prinzip der Autonomie und zusätzlich stehen sie dem Recht auf Nicht-Wissen entgegen.

Zusätzlich stellt sich die Frage der Kostenübernahme: Bei einer verpflichtenden Impfung müssten die Bürger damit rechnen dürfen, dass die Kosten vom Solidarsystem übernommen werden, weil davon auszugehen ist, dass die Kosten relativ hoch sein werden. Da im Gesundheitssystem jedoch schon jetzt über Priorisierung debattiert wird (Schmitz-Luhn und Bohmeier 2013), wird sich die Frage stellen, ob die gesetzliche Krankenversicherung ihre Beiträge erhöhen müsste. Wenn die Kostenübernahme der Krankenversicherung nicht garantiert werden kann und die Impfung deswegen nicht verpflichtend ist, stellt sich die Gerechtigkeitsfrage. Denn nur wenige Reiche werden sich die kostspielige Impfung leisten können. Dieses Dilemma zu lösen, wird eine große Herausforderung werden. 


\section{Recht auf Nicht-Wissen}

Bereits gegenwärtig sind Vortests mittels Biomarkern in Diskussion. Diese sollten zeigen, wer gefährdet ist (Konfliktfall Demenzvorhersage 2018, S. 7). Wichtig ist in diesem Zusammenhang aus ethischer Sicht, dass Menschen, da sie im Falle von Alzheimer Dritte nicht gesundheitlich gefährden, prädiktive Tests freiwillig in Anspruch nehmen können (Konfliktfall Demenzvorhersage 2018, S. 8). Eine Verpflichtung würde dem Recht auf Nicht-Wissen widersprechen, das neben dem Recht auf Wissen gleichrangig zu werten ist (Schöne-Seifert 2007, S. 59). Dieses Recht wäre jedoch wiederum mit der Impfung der gefährdeten Personen nicht vereinbar, weil es dann nicht möglich wäre zu eruieren, bei welchen Personen das Risiko an Alzheimer zu erkranken besteht. Das könnte wiederum Argumenten für die verpflichtende Impfung aller Menschen ab etwa 30 Jahren ohne Vortests Tür und Tor öffnen: Niemand erfährt jemals, ob er ein Alzheimer-Risiko-Patient ist, weil alle von vorn herein gegen die Erkrankung geimpft werden.

Wie sicher die Aussage der Vortests ist, ist eine andere Frage, weil Alzheimer ja vermutlich multifaktoriell entsteht und deshalb auch nach den Vortests diverse Umweltbedingungen auf das Gehirn einwirken könnten, die Alzheimer später entstehen lassen. Die Vorhersagegenauigkeit der derzeit verfügbaren Diagnoseverfahren wird jedenfalls als äußerst gering eingeschätzt. Auch für die Zukunft wird damit gerechnet, dass prädiktive Tests lediglich etwas über das Risiko zu erkranken aussagen können (Konfliktfall Demenzvorhersage 2018, S. 5).

Gesetzt den Fall, man entscheidet sich im Gesundheitssystem für freiwillige prädiktive Tests, stellt sich immer noch die Frage, ob man dann, falls sich laut Test das Risiko an Alzheimer zu erkranken zeigt, geimpft werden möchte. Wird dem Prinzip der Autonomie Rechnung getragen und neben den freiwilligen Vortests wäre auch die Impfung freiwillig, könnte das Wissen bei den Risiko-Personen erhebliche Sorgen und Zukunftsängste hervorrufen. Vor allem in der ersten Zeit der Anwendung der Impfung, in der möglicherweise noch Probleme auftauchen und publik werden: Was, wenn die Impfung bei mir nicht hilft? Was, wenn gerade ich eine Unverträglichkeit gegen den Impfstoff habe und Nebenwirkungen auftreten? Was, wenn es zu Langzeitfolgeschäden kommt, von denen noch niemand wissen kann, weil die Impfung erst seit kurzem eingesetzt wird? Diese möglicherweise auftretenden Fragen können psychisch äußerst belastend sein. Sich dafür zu entscheiden, den prädiktiven Test erst gar nicht in Anspruch zu nehmen, kann allerdings vom Einzelnen auch als schwierig empfunden werden. Denn ,,allein das Wissen um die Möglichkeit des Wissens raubt uns die Unschuld des Nichtwissenkönnens“" (Schöne-Seifert 2007, S. 59).

Wären Diskriminierung und Stigmatisierung jener Menschen, die diese Möglichkeit der Impfung nicht nutzen, zu befürchten, wie auch der Deutsche Ethikrat in Bezug auf Eingriffe in die menschliche Keimbahn zu Bedenken gibt (Deutscher Ethikrat 2019b, S. 24)? Selbst wenn Einigkeit darüber herrschen sollte, dass die Impfung eine freiwillige Option darstellt, könnte der gesellschaftliche Druck dennoch groß sein, sowohl den Vortest als auch dann bei Risiko die Impfung in Anspruch zu nehmen. 
Eine zusätzliche Sorge ist häufig, dass die Gesundheitsdaten in Zeiten der Digitalisierung in falsche Hände geraten und z.B. von Arbeitgebern und Versicherern missbraucht werden könnten (Deutscher Ethikrat 2017). Wenn beispielsweise eine Person laut eines prädiktiven Tests als Risiko-Person für Early Onset Alzheimer feststeht und dies ein Arbeitgeber erfährt, kann es sein, dass aufgrund dieses Risikos der Arbeitsvertrag der Person nicht verlängert wird. Auch Versicherungen (Krankenversicherungen, Lebensversicherungen) könnten Konsequenzen ziehen, wenn sie Gesundheitsdaten ihrer Kunden erhalten, die für sie als Versicherung von finanziellem Nachteil wären (Lemke 2000). Beispiele aus den USA zeigen, dass Ehepaaren die Adoption von Kindern verweigert wird, wenn bei einem Elternteil die Disposition für eine genetische Krankheit vorliegt (Lemke 2000, S. 246-247).

\section{Schwierigkeit der Diagnose}

Es wird immer wieder zugestanden, dass die Alzheimer-Diagnostik eine besondere Herausforderung darstellt (Dubois et al. 2016). Einerseits besteht die Gefahr, dass zu früh eine Demenz diagnostiziert wird, weil von verschiedenen Seiten immer wieder postuliert wird, dass die frühe Diagnose für weitere Planungen und Entscheidungen so wichtig ist, wie etwa das Advance Care Planning (Coors et al. 2015). Bedeutsam ist jedoch, zwischen normaler Altersvergesslichkeit, Mild Cognitive Impairment (MCI) und Alzheimer oder einer anderen Demenz zu unterscheiden. Fest steht, dass die kognitive Leistungsfähigkeit mit zunehmendem Alter abnimmt. Das muss nicht gleich bedeuten, dass es sich um eine Demenz handelt. Wenn aber eine deutliche Verschlechterung des Gedächtnisses über einen längeren Zeitraum bemerkt wird, ist eine Arztkonsultierung angezeigt. MCI wird dann diagnostiziert, wenn eine Person in bestimmten Gedächtnistests deutlich schlechter abschneidet als gleichaltrige (Diehl et al. 2005).

Eine Demenzdiagnose kann eine starke psychische Belastung für die betroffene Person sein. Wenn die Diagnose fälschlicherweise gestellt wurde, ist diese Belastungssituation völlig unnötig. Das zweite Risiko, das die Diagnosestellung mit sich bringt, ist die schwierige Differentialdiagnose zur Depression. In der Frühphase von einer Demenz treten häufig depressive Symptome auf und umgekehrt haben Menschen mit schweren depressiven Störungen häufig auch Gedächtnis- und Merkstörungen, wie sie bei Alzheimer und anderen Demenzen vorkommen (Diehl et al. 2005). Und immer wieder treten Depression und Demenz tatsächlich zusammen auf. Wenn selbst bei aktuellem Vorliegen aller Symptome, die Diagnose AlzheimerDemenz eine Herausforderung darstellt, die mehrerer Absicherungen bedarf (Screenings, Tests, Anamnese, CT, MRT), um zu einer sicheren Diagnose zu gelangen, ist die Frage berechtigt, wie verlässlich prädiktive Tests überhaupt sein können.

\section{Recht auf Krankheit?}

Teilweise wird bereits gegenwärtig besorgt auf Maßnahmen geblickt, die Krankheitsrisiken insgesamt möglichst minimieren sollen: In Rauchverboten, Ernährungsvorgaben und Fitness-Boni sieht etwa Welsh ,eine normative Stoßrichtung der Argumentation, die eine moralische Wertung (oder über Krankenkassen ökonomische 
Benachteiligung) von abweichendem, nicht gesundheitsbewussten Verhalten nahelegt und damit Gefahr läuft, eine Stigmatisierung der Betroffenen zu fördern“ (Welsh 2016, S. 220). Als „Betroffene“ bezeichnet Welsh also Menschen, die rauchen, sich bewusst ungesund ernähren oder keine körperliche Bewegung in ihr Leben integrieren. Sie sieht, auch bei obligatorischen Impfungen, Einschränkungen der Freiheitsrechte (Welsh 2016, S. 232). An dieser Stelle gilt es nachzufragen, ob die Verantwortung des Einzelnen in einer Gesellschaft mit einem solidarischen Gesundheitssystem, in welcher die Mittel knapp sind, höher gewertet werden sollte, wenn er dem System verschuldet (!) zur Last fällt. Solidarität in einem Gesundheitssystem impliziert „das Bereitstellen von Hilfe nach Leistungsfähigkeit bei gleichzeitigem Angebot von Hilfe nach Bedürftigkeit“" (Friedrich 2018, S. 148). Es gibt viele Erkrankungen, die nicht vermeidbar sind, die durch genetische Faktoren verursacht werden oder als Schicksalsschläge, z.B. unverschuldete Arbeits- oder Verkehrsunfälle, zu bezeichnen sind. Das solidarische Gesundheitssystem greift in diesen Situationen aufgrund der daraus folgenden Bedürftigkeit. Und auch bei selbst verschuldeten Unfällen oder bei Unfällen, bei denen nicht völlig nachvollziehbar ist, ob eine Schuld des Betroffenen gegeben ist, wird niemandem die Versorgung verweigert. In diesen Fällen ist es wichtig, dass das Solidarsystem ein gutes Netz zur Verfügung stellt, sodass dem Menschenrecht auf Gesundheit des Einzelnen Rechnung getragen werden kann. Dabei geht es konkret um „das Recht eines jeden auf das für ihn erreichbare Höchstmaß an körperlicher und geistiger Gesundheit" (UN 2000). Dieses Menschenrecht ist eine Errungenschaft. Wird es mit Füßen getreten, wenn man ein Recht auf Krankheit einfordert?

Freilich sind ungesunde Ernährung, mangelnde Bewegung etc. zum Teil auch mit Bildungs- und Informationsmangel verbunden. Zusätzlich werden Lebensweisen innerhalb von Familien tradiert, sodass Kinder nur wenig Chancen haben, die ungesunden Verhaltensweisen ihrer Eltern zu durchbrechen und nicht zu übernehmen, wie Studien belegen (Kurth und Schaffrath Rosario 2007). Deshalb kann neben aufklärerischer Arbeit auch Nudging, also ein kleiner Schubs, der Menschen anstößt, kluge Entscheidungen zu treffen (Thaler und Sunstein 2010), in Erwägung gezogen werden, indem z. B. die ungesunden Lebensmittel deutlich teurer sind als die gesunden.

Wie verhält sich dies aber bei der Impfung gegen Alzheimer? Wenn jedem Bürger das Recht auf Selbstbestimmung zugestanden wird und er sich gegen die Impfung trotz möglichem Risiko entscheidet, dann entscheidet er sich entweder für sein Recht auf Krankheit oder glaubt an sein Schicksal, in das er nicht eingreifen möchte. Auch religiöse Überzeugungen oder die Sorge vor schlimmeren Erkrankungen durch die Impfung können hier eine wesentliche Rolle spielen, wie auch die aktuelle Impfdebatte zeigt. Die Aufklärung über die Sinnhaftigkeit einer Impfung müsste mittels Kampagnen, wie sie schon jetzt der Deutsche Ethikrat zum Thema Impfen allgemein vorschlägt, stattfinden. Zwang zur Impfung scheint hingegen aufgrund der unterschiedlichen Zugänge, Sorgen und Lebensformen der Menschen problematisch. 


\section{Schlussüberlegungen}

Ökonomische Aspekte im solidarischen Gesundheitssystem zu bedenken ist wichtig, das steht außer Zweifel. Letztlich dürfen diese aber nicht allein über Gesundheitsentscheidungen einzelner Personen stehen. In einer Gesellschaft, in welcher der Einzelne ein Recht auf Autonomie hat, scheint es aus ethischer Sicht problematisch, paternalistisch in das individuelle Leben einzugreifen. Wichtig werden dennoch Aufklärungskampagnen sein, um zu informieren und die Bürger zu befähigen, die für sie individuell richtige Entscheidung zu treffen. Damit ist auch verbunden, sich gegen prädiktive Tests und gegen eine Alzheimer-Impfung entscheiden zu können. Das bedeutet, dass die Gesellschaft weiterhin Pflege- und Betreuungsmöglichkeiten für Menschen mit Alzheimer zur Verfügung stellen muss, damit die Möglichkeit der Wahl gegeben ist. Pflegebedürftige Menschen mit Alzheimer, anderen Demenzen und auch kognitiv gesunde, aber körperlich beeinträchtigte ältere Menschen wird es immer geben. Die Aufgabe einer solidarischen Gesellschaft ist es, für diese Menschen in vulnerablen Situationen gut zu sorgen.

Eine ganz andere hypothetische Frage ist, wie es um die Atmosphäre einer Gesellschaft bestellt ist, wenn sich niemand mehr um Alte und Kranke kümmern will, weil für alles, was eine Last bedeuten könnte, eine „Lösung“ gefunden wird. In dieser Dystopie werden Menschen mit Behinderung dank PID und PND erst gar nicht mehr geboren, Menschen, die unheilbar krank sind, legt man nahe, vom ärztlich assistierten Suizid Gebrauch zu machen, und Menschen, die sich nicht gegen Alzheimer impfen lassen, werden als verantwortungslos angesehen, weil durch sie ein „Aufwand“ in personeller und finanzieller Hinsicht entstehen kann. Dass Überlegungen dieser Art aber nicht lediglich graue Theorie sind, sondern von manchen Politikern tatsächlich in Betracht gezogen werden, soll abschließend ein Beispiel aus Bulgarien zeigen: Im Jahr 2008 sorgte die bulgarische Sozialministerin Emilia Maslarova für Aufsehen, als sie vorschlug, post-natale Behinderungen bei Kindern möglichst vorgeburtlich zu verhindern. Um dies durchzusetzen, empfahl sie Strafen für Frauen, die sich der dafür erforderlichen Untersuchungen in den ersten drei Monaten der Schwangerschaft nicht unterziehen, und bei Geburt des behinderten Kindes, Kürzungen des Kindergeldes (Karamelska 2010, S. 114f.). Von Seiten bulgarischer Intellektueller, wie Kalin Yanakiev, wurde dies sehr kritisch gesehen. Yanakiev monierte, dass die Sozialministerin versuche das Leid zu beseitigen, indem sie das Mitleid zerstört (Karamelska 2010, S. 115).

Wie wir in unserer Gesellschaft die Alzheimer-Impfung einsetzen wollen, wenn es zum Durchbruch in der Forschung kommt, muss dann zum gegebenen Zeitpunkt im Lichte der aktuellen Situation des Gesundheitssystems aus ethischer Sicht analysiert werden. Der vorliegende Text hat bereits einige hypothetische Vorüberlegungen angestellt, die darauf verweisen, dass eine Impfung gegen Alzheimer mit vielen ethischen Herausforderungen verbunden sein wird.

Funding Open access funding provided by University of Graz.

Open Access Dieser Artikel wird unter der Creative Commons Namensnennung 4.0 International Lizenz veröffentlicht, welche die Nutzung, Vervielfältigung, Bearbeitung, Verbreitung und Wiedergabe in jeglichem Medium und Format erlaubt, sofern Sie den/die ursprünglichen Autor(en) und die Quelle ord- 
nungsgemäß nennen, einen Link zur Creative Commons Lizenz beifügen und angeben, ob Änderungen vorgenommen wurden.

Die in diesem Artikel enthaltenen Bilder und sonstiges Drittmaterial unterliegen ebenfalls der genannten Creative Commons Lizenz, sofern sich aus der Abbildungslegende nichts anderes ergibt. Sofern das betreffende Material nicht unter der genannten Creative Commons Lizenz steht und die betreffende Handlung nicht nach gesetzlichen Vorschriften erlaubt ist, ist für die oben aufgeführten Weiterverwendungen des Materials die Einwilligung des jeweiligen Rechteinhabers einzuholen.

Weitere Details zur Lizenz entnehmen Sie bitte der Lizenzinformation auf http://creativecommons.org/ licenses/by/4.0/deed.de.

\section{Einhaltung ethischer Richtlinien}

Interessenkonflikt M. Schmidhuber gibt an, dass kein Interessenkonflikt besteht.

Ethische Standards Für diesen Beitrag wurden von der Autorin keine Studien an Menschen oder Tieren durchgeführt. Für die aufgeführten Studien gelten die jeweils dort angegebenen ethischen Richtlinien.

\section{Literatur}

Alzheimer's Disease International (2010) World Alzheimer Report 2010. The global economic impact of dementia. Alzheimer's Disease International, London

Alzheimer's Disease International (2018) World Alzheimer Report 2018. The state of the art of dementia research: New frontiers. Alzheimer's Disease International, London

Bachmann MF, Jennings GT, Vogel M (2018) A vaccine against Alzheimer's disease: anything left but faith? Expert Opin Biol Ther 19(1):73-78

Bozzaro C, Krause F, Weismann M (2019) Uterustransplantation. Ethisch gerechtfertigt? Ethik Med 31(2):113-129

Coors M, Jox R, in der Schmitten J (2015) Advance Care Planning. Von der Patientenverfügung zur gesundheitlichen Vorausplanung. Kohlhammer, Stuttgart

Demicheli V, Debalini MG, Rivetti A (2009) Vaccines for preventing tick-borne encephalitis. Cochrane Database Syst Rev 21(1):CD977. https://doi.org/10.1002/14651858.CD000977.pub2

Deutscher Ethikrat (2017) Big Data und Gesundheit - Datensouveränität als informationelle Freiheitsgestaltung. Stellungnahme. Deutscher Ethikrat, Berlin

Deutscher Ethikrat (2019a) Impfen als Pflicht? Stellungnahme. Deutscher Ethikrat, Berlin

Deutscher Ethikrat (2019b) Eingriffe in die menschliche Keimbahn. Stellungnahme. Deutscher Ethikrat, Berlin

Diehl J, Förstl H, Kurz A (2005) Alzheimer-Krankheit. Symptomatik, Diagnose und Therapie. Z Med Ethik 51(1):3-12

Dubois B, Padovani A, Scheltens P, Rossi A, Dell'Agnello G (2016) Timely diagnosis for Alzheimer's disease: a literature review on benefits and challenges. J Alzheimers Dis 49(3):617-631

Friedrich DR (2018) Rechtfertigung solidarischer Gesundheitsversorgung. Warum Mitbestimmung gut für unsere Gesundheit ist. Mentis, Münster

Gräßel E, Behrndt E (2016) Belastungen und Entlastungsangebote für pflegende Angehörige. In: Jacobs K, Kuhlmey A, Greß S, Klauber J, Schwinger A (Hrsg) Pflege-Report 2016. Schattauer, Stuttgart, S 168-187

Gronemeyer R (2013) Das 4. Lebensalter. Demenz ist keine Krankheit. Pattloch, München

Hirte M (2012) Impfen. Pro \& Contra. Das Handbuch für die individuelle Impfentscheidung. Knaur, München

Karamelska T (2010) Bioethische Fragen in Bulgarien aus orthodoxer Sicht. In: Voigt F (Hrsg) Religion in bioethischen Diskursen. Interdisziplinäre, internationale und interreligiöse Perspektiven. De Gruyter, Berlin, S 103-131

Konflikfall Demenzvorhersage (2018) Gemeinsame Stellungnahme der Stakeholder-Konferenz im Rahmen des Diskursverfahrens „Konfliktfall Demenzvorhersage“. Konflikfall Demenzvorhersage, Göttingen 
Kurth BM, Schaffrath Rosario A (2007) Die Verbreitung von Übergewicht und Adipositas bei Kindern und Jugendlichen in Deutschland. Ergebnisse des bundesweiten Kinder- und Jugendgesundheitssurveys (KiGGS). Bundesgesundheitsblatt Gesundheitsforschung Gesundheitsschutz 50:736-743

Lemke T (2000) Die Regierung der Risiken. Von der Eugenik zur genetischen Gouvernementalität. In: Bröckling U, Krasmann S, Lemke T (Hrsg) Gouvernementalität der Gegenwart. Studien zur Ökonomisierung des Sozialen. Suhrkamp, Frankfurt/Main, S 227-264

Lenzen-Schulte M (2018) Antidementiva scheitern reihenweise. Dtsch Arztebl 115(5):A200-A202

Robert Koch Institut (2019) Epidemiologisches Bulletin Nr. 34. https://www.rki.de/DE/Content/Infekt/ EpidBull/Archiv/2019/Ausgaben/34_19.pdf?_blob=publicationFile. Zugegriffen: 7. Okt. 2019

Schmietow B, Eberbach W, Kaulich M (2019) Gene Editing in der Krebsforschung: technische, ethische und rechtliche Aspekte. Onkologe. https://link.springer.com/article/10.1007\%2Fs00761-019-05999. Zugegriffen: 7. Okt. 2019

Schmitz-Luhn B, Bohmeier A (Hrsg) (2013) Priorisierung in der Medizin. Kriterien im Dialog. Springer, Berlin

Schöne-Seifert B (2007) Grundlagen der Medizinethik. Kröner, Stuttgart

Thaler RH, Sunstein CR (2010) Nudge. Wie man kluge Entscheidungen anstößt, 7. Aufl. Econ, Berlin

Tse KH, Herrup K (2017) Re-imaging Alzheimer's disease-the diminishing importance of amyloid and a glimpse of what lies ahead. J Neurochem 143:432-444

UN-Ausschuss für wirtschaftliche, soziale und kulturelle Rechte (2000) Allgemeine Bemerkung Nr. 14: Das Recht auf ein Höchstmaß an Gesundheit (Artikel 12) UN-Dok. CESCR E/C.12/2000/4, 11.08.2000, Ziffer 12, b), i)

Wallesch CW, Förstl H (2017) Demenzen. 3. Aufl. Thieme, Stuttgart

Welsh C (2016) Brauchen wir ein Recht auf Krankheit? Historische und theoretische Überlegungen im Anschluss an Juli Zehs Roman Corpus Delicti. In: Frewer A, Bielefeldt H (Hrsg) Das Menschenrecht auf Gesundheit. transcript, Bielefeld, S 215-238

World Health Organization (2019) Vaccines and immunization. http://www.euro.who.int/en/health-topics/ disease-prevention/vaccines-and-immunization/publications/surveillance-and-data/measles-andrubella-elimination-country-profiles/2019. Zugegriffen: 7. Okt. 2019

World Health Organization (1986) Ottawa-Charta zur Gesundheitsförderung. WHO, Genf

Zeltins A et al (2017) Incorporation of tetanus-epitope into virus-like particles achieves vaccine responses even in older recipients in models of psoriasis, Alzheimer's and cat allergy. Npj Vaccines. https:// www.nature.com/articles/s41541-017-0030-8.pdf. Zugegriffen: 7. Okt. 2019

Zentrum der Gesundheit (2019) Impfschaden: Meldung, Anerkennung, Entschädigung. https://www. zentrum-der-gesundheit.de/impfschaden.html. Zugegriffen: 7. Okt. 2019 